\title{
Approximate SER for M-PSK using MRC and STTD Techniques over Fading Channels
}

\author{
Mahmoud A. Khodeir \\ Department of Electrical Engineering \\ Jordan University of Science and Technology \\ Irbid, Jordan. P.O. Box 3030
}

\author{
Muteeah A. Jawarneh \\ Department of Electrical Engineering \\ Jordan University of Science and Technology \\ Irbid, Jordan. P.O. Box 3030
}

\begin{abstract}
In this study, approximate symbol error rate (SER) expressions for M-ary phase shift keying (M-PSK) modulation scheme over independent and identically distributed (i.i.d) slow-flat Rician and Rayleigh fading channels are derived. Simulation results show the superior impact of using the maximum ratio combining (MRC) space diversity technique on the overall performance. In particular, the communication reliability (i.e., capacity and coverage) will increase by increasing the diversity order (i.e., the number of the combiner's branches), where less power is needed to achieve the same probability of error. Then, a comparison between the approximate and exact probability of symbol error is performed and the results are shown to be comparable (1-2 dB). Next, approximate SER expression is derived over i.i.d slow-flat Nakagami- $m$ fading channels. In particular, space time transmit diversity (STTD) technique is used to enhance the reliability of the proposed model using two transmit antennas and one receive antenna. The simulation results show the effect of the Nakagami- $m$ parameter, $m$, on the SER where the performance will improve by increasing the value of $m$ where fading is less severe in this case. Furthermore, the performance of the SER is lower for higher values of SNR and is worse for high order PSK modulation schemes.
\end{abstract}

Keywords-Rician fading channel; Rayleigh fading channel; Nakagami-m fading channel; maximum ratio combining; space diversity; space time transmit diversity; symbol error rate.

\section{INTRODUCTION}

In general, the overall performance of any wireless communication system is severely degraded by the timevarying multi-path fading which make the analysis relatively difficult. In particular, fading results from the interference between two or more versions of the transmitted signals that arrive at the receiver with slightly different delay times. Time diversity, frequency diversity, and space diversity are the main diversity techniques that can efficiently be deployed to overcome the destructive effects of fading.

Generally, space diversity deploys multiple antennas either at the transmitting end and/or at the receiving end to get advantage of the diversity gain in order to mitigate the multipath fading in an effective way. Here, multiple antennas provide the receiver with several observations of the same signal. Hence, each antenna will experience a different interference environment, and therefore, if one antenna is experiencing a deep fading most probable the other antennas have adequate signals. Namely, MRC is a classical combining technique in which the signals from the received antenna elements are weighted such that the signal to noise ratio (SNR) achieved due to the total sum of the received signals is maximized. Moreover, the distance between the multiple antennas must be selected to be sufficiently far apart to guarantee that signals from different antennas face uncorrelated fading process.

First of all, the moment generating function (MGF) of the received SNR was used in [1] to analyze the error rate performance for M-PSK modulation scheme, where independent phase estimation was assumed over Rician, Rayleigh, and Nakagami- $m$ fading channels. Next, the authors in [2] have derived the exact bit error rate (BER) for a binary phase shift keying (BPSK) modulation scheme in a synchronous cochannel interference (CCI) under Nakagami-m flat fading channel using both of equal gain combining (EGC) technique with characteristic function (CF) method and selection combining (SC) technique with Fourier series method. Furthermore, the authors in [3] studied the exact BER performance for BPSK and binary frequency shift keying (BFSK) modulation schemes over MIMO system were derived for i.i.d slow-flat Nakagami- $m$ fading channel. Here, the MGF based analytical method was used to analyze this model using both transmit and receive antenna selection (TAS/RAS).

Moreover, the outage and BER analysis of the MRC for band-limited BPSK system with micro-cellular system over i.i.d Rayleigh and Nakagami- $m$ slow-flat fading channels were presented in [4]. Here, the authors considered an independent fading gain and asynchronous timing as well as co-channel interference in the derivation to obtain the exact closed-form outage probability expression with both of equal and unequal interferer's power. Next, both of the optimum and suboptimum diversity combining were derived in [5] for differential and coherent signals with M-PSK modulation scheme over Rician fading channels plus the impulsive classA noise. Here, the tight performance is upper-bounded for both of the combining schemes and the derivations were done for both MRC and EGC diversity techniques. In particular, a relatively large performance gain was shown for both of the suboptimum noncoherent combining (SNS) and the suboptimum coherent combining (SCC) schemes.

Next, the approximate BER for M-ary quadrature shift keying (M-QAM) have been derived in [6] over i.i.d slow-flat Rician fading channel, where a single exponential function was used to approximate the BER for M-QAM modulation scheme over a Gaussian channel. Furthermore, the average BER for band-limited BPSK over i.i.d Nakagami- $m$ slow-flat was derived in [7] where the authors assumed a synchronous 
timing and independent fading gain, equal gain, and maximum desired user power selection combining. Moreover, the cochannel interference was considered, and the characteristic function and Fourier series methods for different values of the diversity order and fading parameter were used in the derivation. Therefore, the performance and the superiority were increased when the diversity order was increased. Finally, the authors in [8] derived the BER for non-coherent fast-frequency-hopped M-ary orthogonal frequency shift keying (FFH/MFSK) for non-selective Nakagami- $m$ slow-flat fading channel, where the CF based approach was used. Furthermore, the authors in [9] derived an exact expression for the BER for M-PSK and M-QAM under Nakagami- $m$ fading channels with STTD. This expression was obtained numerically and the results showed a better BER performance when both of the SNR and the parameter $m$ were increased.

In this paper, an approximate expression of the SER for MPSK modulation over a Gaussian channel is used to derive the approximate expressions for the SER for M-PSK over i.i.d slow-flat Rician and Rayleigh fading channels using MRC space diversity technique. In particular, the MRC space diversity is used to enhance the overall coverage and the capacity of the proposed model. Here, the SER for optimum MRC receives diversity with channel side information known at the receiver (CSIR) is acquired. Moreover, the proposed approximation will be bounded within $1 \mathrm{~dB}$ for signal alphabet $M \geq 4$ and for $0 \leq \mathrm{SNR} \leq 30 \mathrm{~dB}$. Next, the approximate expression for the SER for M-PSK over i.i.d slow-flat Nakagami- $m$ fading channels is derived using STTD technique to improve the overall performance. In general, STTD technique is applied using two transmit antennas and one receive antenna. Overall, the rest of this paper is organized as follows. In section II, approximate SER expressions for MPSK over i.i.d Rician and Rayleigh fading channels are derived. Then approximate SER expressions for the M-PSK using MRC diversity are obtained in Section III. Next, in Section IV an approximate SER for M-PSK using STTD over i.i.d Nakagami- $m$ fading channels is derived. Finally, Sections $\mathrm{V}$ and VI present the simulation results analysis and the conclusion.

\section{APPROXIMATE SER FOR M-PSK OVER RICIAN FADING CHANNELS}

A communication system with M-PSK signaling over AWGN plus i.i.d slow-flat Rician fading channel is considered. Here, the transmitted signal is perturbed by AWGN with two-sided power spectral density $N_{0} / 2$. Moreover, the effect of the Rician fading channel on the signal is multiplicatively distorted by the Rician parameter $k$. The SER of the proposed model is given by [10]:

$$
P_{S}=2 Q\left(\sqrt{2 \gamma_{S}} \sin (\pi / M)\right)
$$

where $\gamma_{S}=E_{S} / N_{0}$ represents the SNR per symbol, $E_{S}$ is the overall symbol energy, $M$ is the number of modulation levels, and $Q$ (.) is the $Q$-function which is approximated by [11]:

$$
Q(x) \approx 0.5 \exp \left(-x^{2} / 2\right)
$$

Therefore, the probability of symbol error for M-PSK over a Gaussian channel can be rewritten as follows:

$$
P_{S}=\exp \left(-\sin ^{2}(\pi / M) \gamma \log _{2}(M)\right)
$$

where $\gamma_{s}=\gamma_{b} \log _{2}(M)=\gamma \log _{2}(M)$. Here, $\gamma_{b}$ represents the SNR per bit. Moreover, the SER expression, which is caused by stationary AWGN terms, depends only on the instantaneous SNR associated with each symbol. Furthermore, the instantaneous SNR per symbol becomes a random variable due to fading effects. Therefore, by using the parameter $k$ and applying change of variables, the pdf of the instantaneous SNR, $\gamma$, is given by [6]:

$$
\begin{aligned}
p_{\gamma}(\gamma)= & (1+k) \frac{\exp [-k]}{\bar{\gamma}} \exp \left[\frac{-(1+k) \gamma}{\bar{\gamma}}\right] \\
& \times I_{o}\left(2 \sqrt{\frac{k(1+k) \gamma}{\bar{\gamma}}}\right)
\end{aligned}
$$

where $\bar{\gamma}$ represents $E(\gamma)$. Furthermore, the probability of symbol error, $P_{1}(E)$, can be found by taking the average of the conditional probability of error, $P(E / \gamma)$, over the pdf of $\gamma$ as follows:

$$
P_{1}(E)=\int_{0}^{\infty} P(E / \gamma) p_{\gamma}(\gamma) \mathrm{d} \gamma
$$

The above equation can be rewritten after some manipulations as follows [13]:

$$
\begin{aligned}
& P_{1}(E) \approx \frac{(1+k) \exp (-k)}{(1+k)+\left(\sin ^{2}(\pi / M) \log _{2}(M) \bar{\gamma}\right)} \\
& \quad \times \exp \left(\frac{k(1+k)}{(1+k)+\left(\sin ^{2}(\pi / M) \log _{2}(M) \bar{\gamma}\right)}\right)
\end{aligned}
$$

Finally, by substituting $k=0$ in (8), one gets the approximate SER expression for M-PSK over Rayleigh fading channel.

\section{ANALYSIS FOR M-PSK RICIAN LINEAR FADING Channel With MRC SPACE DiVERSITY}

The instantaneous SNR at the output of the MRC combiner is the sum of the instantaneous SNR at each individual branch (i.e., $\gamma=\sum_{i=1}^{N} \gamma_{i}$ ) where $N$ is the number of the combiner's branches. Then, the new pdf of $\gamma$ after applying diversity combining is given by [6]:

$$
\begin{gathered}
p_{\gamma(\gamma)}=\left(\frac{N+k}{\bar{\gamma}}\right)\left[\frac{(N+k) \gamma}{k \bar{\gamma}}\right]^{\frac{N-1}{2}} \exp \left(\frac{-(N+k) \gamma+k \bar{\gamma}}{\bar{\gamma}}\right) \\
I_{N-1}\left(2 \sqrt{\frac{k(N+k) \gamma}{\bar{\gamma}}}\right)
\end{gathered}
$$

where $k=\sum_{i=1}^{N} k_{i}$ and $I_{N}($.$) is the n$-th order modified Bessel function of the first kind. Here, the error probability is a function of $k, \bar{\gamma}$, and $N$. Next, by averaging the conditional probability of error over the pdf of $\gamma$, one gets [13]: 


$$
\begin{aligned}
P_{2}(E) & \approx \int_{0}^{\infty}\left(\frac{N+k}{\bar{\gamma}}\right)\left[\frac{(N+k)}{k \bar{\gamma}}\right]^{\frac{N-1}{2}} \gamma^{\left(\frac{N-1}{2}\right)} \exp \left(\frac{-(N+k) \gamma}{\bar{\gamma}}\right) \\
& \times \exp (-k) I_{N-1}\left(2 \sqrt{\frac{k(N+k) \gamma}{\bar{\gamma}}}\right) \\
& \times \exp \left(-\sin ^{2}(\pi / M) \gamma \log _{2}(M)\right) d \gamma
\end{aligned}
$$

After some manipulations and simplifications, one can get the following expression for the probability of error [13]:

$$
\begin{gathered}
P_{2}(E) \approx\left[\frac{(N+k)+\left(\sin ^{2}(\pi / M) \bar{\gamma} \log _{2}(M)\right.}{\bar{\gamma}}\right]^{-N} \\
\times \exp \left(\frac{k(N+k)}{\left.(N+K)+\sin ^{2}(\pi / M) \bar{\gamma} \log _{2}(M)\right)}\right) \\
\quad \times \\
\exp (-k)\left(\frac{N+k}{\bar{\gamma}}\right) N
\end{gathered}
$$

Here, when the value of $k$ is set to zero, one can get the approximate probability of error for M-PSK over i.i.d Rayleigh fading channel using $N$ branches which is given by:

$$
P_{2}(E)=\left[\frac{N+\sin ^{2}(\pi / M) \bar{\gamma} \log _{2}(M)}{\bar{\gamma}}\right]^{-N}\left(\frac{N}{\bar{\gamma}}\right)^{N}
$$

\section{APPROXIMATE SER FOR M-PSK WITH STTD OVER NAKAGAMI-M FADING CHANNEL}

In general, STTD is an open loop technique which is adopted by the 3GPP to increases the diversity gain. Considering a downlink wireless channel with two transmit antennas and one receive antenna as shown in Fig. 1 [9]:

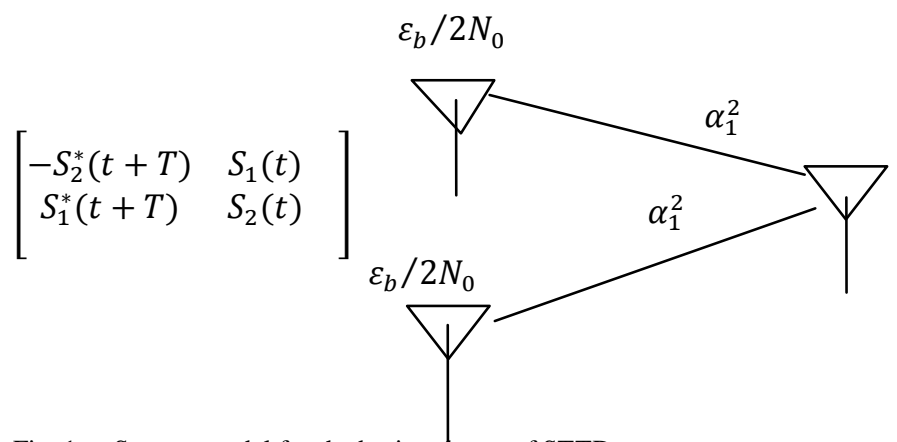

Fig. 1. System model for the basic scheme of STTD

The average transmits SNR per bit for each antenna in STTD technique model is denoted by $\varepsilon_{b} / 2 N_{O}$. This assumption is valid to get the same total radiated power similar to the case with one transmit antenna. Here, $\alpha_{1}{ }^{2}$ and $\alpha_{2}{ }^{2}$ denote the independent channel gains (i.e., square of the fading channel factor), $S_{I}(t)$ and $S_{2}(t)$ are the symbols transmitted by both antennas at time $t,-S_{2}{ }^{*}(t+T)$ and $S_{1}{ }^{*}(t+T)$ at time $t+T$, where ${ }^{*}$ denotes the complex conjugate operation. The Nakagami- $m$ parameter, $m$, characterizes the severity of the signal fading. In particular, $m=0.5$ represents the onesided Gaussian fading channel and $m=1$ describes the Rayleigh fading channel. Furthermore, the lognormal and Rician distributions can be approximated by the Nakagami- $m$ distribution when $m>1$. Now, the pdf of the Nakagami- $m$ distribution is given by [9]:

$$
p_{\alpha}(\alpha)=\frac{2}{\Gamma(m)}\left(\frac{m}{\Omega}\right)^{m} \alpha^{2 m-1} e^{-m \alpha^{2} / \Omega}
$$

where $\Omega=E\left(\alpha^{2}\right)$ represents the average channel gain, $\Gamma($.$) is the gamma function, m=\frac{\Omega^{2}}{E\left[\left(R^{2}-\Omega^{2}\right)\right]}$ and $E\left(R^{n}\right)$ is given by:

$$
E\left(R^{n}\right)=\frac{\Gamma\left(m+\frac{1}{2} n\right)}{\Gamma(m)}\left(\frac{\Omega}{m}\right) n / 2 \quad, m \geq 0.5
$$

Now, let $\gamma_{b}=\alpha^{2} \frac{\varepsilon_{b}}{N_{o}}$, then one can write the pdf of the received SNR per bit for i.i.d Nakagami- $m$ flat fading channels as follows:

$$
p_{\gamma_{b}}\left(\gamma_{b}\right)=\frac{m^{m}}{\bar{\gamma}_{b}^{m} \Gamma(m)} \gamma_{b}^{m-1} e^{-m \gamma_{b} / \bar{\gamma}_{b}}
$$

where $\bar{\gamma}_{b}=E\left(\alpha^{2}\right) \frac{\varepsilon_{b}}{N_{O}}$ represents the average received SNR. Now, the channel gain with STTD diversity technique over i.i.d Nakagami- $m$ flat fading channel is given by [9]:

let

$$
\alpha^{2}=\frac{1}{2}\left(\alpha_{1}^{2}+\alpha_{2}^{2}\right)
$$

$$
y=\alpha^{2}, x_{1}=\alpha_{1}^{2}, \quad x_{2}=\alpha_{2}^{2}
$$

Therefore, $\frac{\varepsilon_{b}}{N_{0}}=\frac{\bar{\gamma}_{b}}{\Omega}$ and the pdf of $y$ can be rewritten [6]:

$$
p_{y}(y)=2 \sqrt{\pi}\left(\frac{m}{\Omega}\right)^{2 m} \frac{y^{2 m-1}}{\Gamma(m) \Gamma\left(\frac{1}{2}+m\right)} e^{-2 m y / \Omega}
$$

Hence, the pdf of the received SNR with STTD technique over i.i.d Nakagami- $m$ flat fading channel is given by [9]:

$$
\begin{aligned}
p_{\gamma}(\gamma) & =\frac{1}{\varepsilon_{b} / N_{0}} p_{\gamma}\left(\frac{\gamma_{b}}{\varepsilon_{b} / N_{0}}\right) \\
& =\frac{2 \sqrt{\pi} m^{2 m} \gamma_{b}{ }^{2 m-1}}{\Gamma(m) \Gamma(1 / 2+m) \bar{\gamma}_{b}{ }^{2 m}} \exp \left(\frac{-2 m \gamma_{b}}{\bar{\gamma}_{b}}\right)
\end{aligned}
$$

The pdf of $\gamma$ at the received SNR per bit for i.i.d Nakagami- $m$ fading channel with STTD technique using two transmit antennas and one receive antenna is given as follows [9]:

$$
p_{\gamma}(\gamma)=\frac{2 \sqrt{\pi} m^{2 m} \gamma^{2 m-1}}{\Gamma(m) \Gamma(1 / 2+m) \bar{\gamma}^{2 m}} \times \exp \left(\frac{-2 m \gamma}{\gamma}\right)
$$

Furthermore, by using the approximation of the probability of symbol error for M-PSK over a Gaussian channel which is shown in (3), one can find the probability of symbol error, $P_{3}(E)$, using (6) as follows:

$$
\begin{aligned}
P_{3}(E) \approx \int_{0}^{\infty} \exp & \left(-\sin ^{2}(\pi / M) \gamma \log _{2}(M)\right) \\
& \times \exp \left(\frac{-2 m \gamma}{\bar{\gamma}}\right) \frac{2 \sqrt{\pi} m^{2 m} \gamma^{2 m-1}}{\Gamma(m) \Gamma(1 / 2+m) \bar{\gamma}^{2 m}} d \gamma
\end{aligned}
$$

which can be written as follows? 


$$
\begin{array}{r}
P_{3}(E) \approx \frac{2 \sqrt{\pi} m^{2 m}}{\Gamma(m) \Gamma(1 / 2+m) \bar{\gamma}^{2 m}} \int_{0}^{\infty} \gamma^{2 m-1} \\
\times \exp \left[-\left(\sin ^{2}(\pi / M) \gamma \log _{2}(M)\right)+\frac{-2 m \gamma}{\bar{\gamma}}\right] d \gamma
\end{array}
$$

Now, to simplify the calculations let:

$$
C=\frac{2 \sqrt{\pi} m^{2 m}}{\Gamma(m) \Gamma(1 / 2+m) \bar{\gamma}^{2 m}}
$$

Then (20) can be rewritten as follows:

$P_{3}(E)=$

$C \int_{0}^{\infty} \exp \left[\frac{\left.-\left(\sin ^{2}(\pi / M) \gamma \log _{2}(M)\right) \bar{\gamma}\right)-2 m \gamma}{\bar{\gamma}}\right] \gamma^{2 m-1} d \gamma$

Next, to solve (21), one can use the following integral [12]:

$$
\int_{0}^{\infty} x^{n} e^{-x a} d x=\left[\begin{array}{cr}
\frac{\Gamma(n+1)}{a^{n+1}} & n>-1, a>0 \\
\frac{n !}{a^{n+1}} & a>0, n \text { :positive }
\end{array}\right]
$$

Now let

$$
\begin{gathered}
n=2 m-1, \\
a=\frac{\sin ^{2}(\pi / M) \log _{2}(M) \bar{\gamma}+2 m}{\bar{\gamma}}
\end{gathered}
$$

Then (21) can be written for the case when $n>-1, a>0$, as follows:

$$
\begin{aligned}
P_{3}(E) & \approx C_{2} \times \frac{\Gamma(n+1)}{a^{n+1}} \approx C_{2} \times \frac{\Gamma(2 m-1+1)}{\left[\frac{\sin ^{2}(\pi / M) \log _{2}(M) \bar{\gamma}+2 m}{\bar{\gamma}}\right]^{2 m}} \\
& \approx \frac{2 \sqrt{\pi} m^{2 m}}{\Gamma(m) \Gamma(1 / 2+m) \bar{\gamma}^{2 m}} \times \frac{\Gamma(2 m)}{\left[\frac{\sin ^{2}(\pi / M) \log _{2}(M) \bar{\gamma}+2 m}{\bar{\gamma}}\right]^{2 m}}
\end{aligned}
$$

Next, the above equation can be rewritten as follows:

$$
\begin{aligned}
& P_{3}(E) \approx \\
& \frac{2 \sqrt{\pi} \Gamma(2 m)}{\Gamma(m) \Gamma(1 / 2+m)} \times \frac{m^{2 m}}{\left[\sin ^{2}(\pi / M) \log _{2}(M) \bar{\gamma}+2 m\right]^{2 m}}
\end{aligned}
$$

Furthermore, when $a>0$ and $n$ is positive, one can write (21) as follows:

$$
\begin{aligned}
& P_{3}(E) \\
& \frac{2 \sqrt{\pi} m^{2 m}}{\Gamma(m) \Gamma(1 / 2+m)} \frac{(2 m-1) !}{\left[\sin ^{2}(\pi / M) \log _{2}(M) \bar{\gamma}+2 m\right]^{2 m}}
\end{aligned}
$$

\section{NUMERICAL RESUlTS}

Here, the simulation results for the approximate and exact SER for Rician, Rayleigh, and Nakagami- $m$ channels with MRC and STTD diversity techniques are presented. First of all, Fig. 2 shows the approximate SER over Rician fading channel with MRC diversity technique for $M=8$ and 16. Here, when $N=2$ and for different values of the Rician parameter, $k$, one can see the direct impact of increasing $k$ on improving the overall performance. However, the worst case (i.e., the Rayleigh fading channel case) is achieved when $k=0$. Furthermore, when $N=4$, one can see the great reduction in the average SNR as shown in Fig. 3. The same approach is applied in Figs 4 and 5 for $M=16$ and 32. The overall performance is improved by applying diversity. However, when $M$ increases the probability of error increases (i.e., the receiver needs more power to achieve the same probability of error). Next, Figs. 6, 7 and 8 show comparisons between the approximate and the exact SER values over the Rayleigh fading channels using MRC technique for $M=8,16$ and 64, respectively. Here, one can observe the overall significant improvement of the SER in the proposed model by using MRC diversity. In particular, when the diversity order increases the overall performance is improved.

Now, in Fig. 9 shows the approximate probabilities of error over Rayleigh fading channel with MRC diversity technique for $M=8,16$, and 64 when $N=1,4$, and 10 . Here, one can note that the overall performance when $M=8$ or 16 is better than the case when $M=64$. Next, in Fig. 10, the approximate SER for $M$-PSK with STTD is shown over Nakagami- $m$ fading channels when $M=4$ for different values of the parameter $m$. The direct impact of increasing $m$ on improving the overall performance is obvious (i.e., for $m=3$, one can see the great reduction in the average SNR). However, one can get the worst case scenario when $m<1$. The same approach is applied in Figs. 11 and 12 for $M=16$ and 32 . Again, the probability of error is increased for the higher order of $M$.

Furthermore, Fig. 13 show comparison between the approximate SERs for M-PSK modulation scheme under Nakagami- $m$ fading channel with STTD diversity technique for different values of $M$. In particular, the comparison is done for $M=4,16$, and 32 when $m=1$ and $m=6$. From the simulation results, one can observe again the effect of increasing the parameter $m$ on the SER. The overall performance of the model is great when $m=6$ as compared with the Rayleigh fading channel case (i.e., when $m=1$ ). Finally, Fig. 17 shows comparisons between the approximate SER for different values of $M$ (e.g., $M=4,8$, and 16) when $m$ $=6,3$, and 0.5 , respectively. Again, one can note the overall performance under Nakagami- $m$ fading channel when $m=6$ or 3 outperforms the case when $m=0.5$.

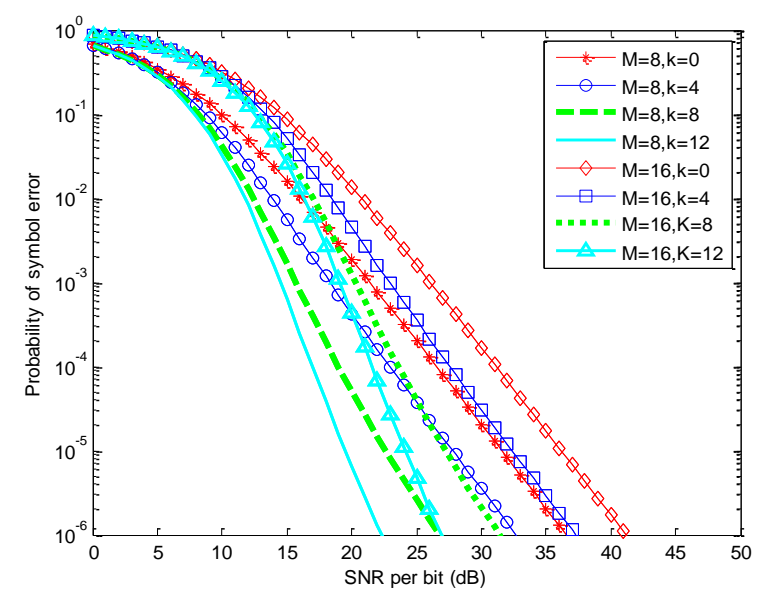

Fig. 2. Approximate SER for M-PSK over Rician fading channels for $N=2$ and for various values of $k$ when $M=8,16$. 


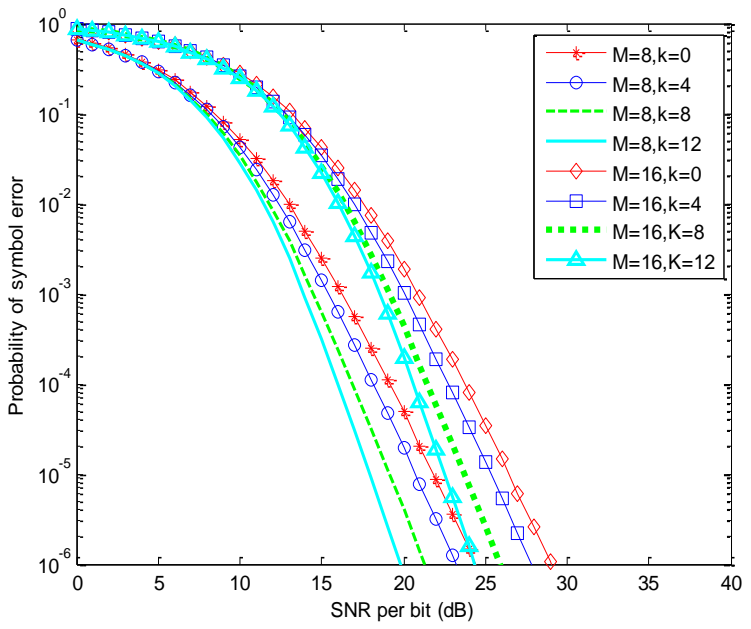

Fig. 3. Approximate SER for M-PSK over Rician fading channels for $N=4$ and for various values of $k$ when $M=8,16$.

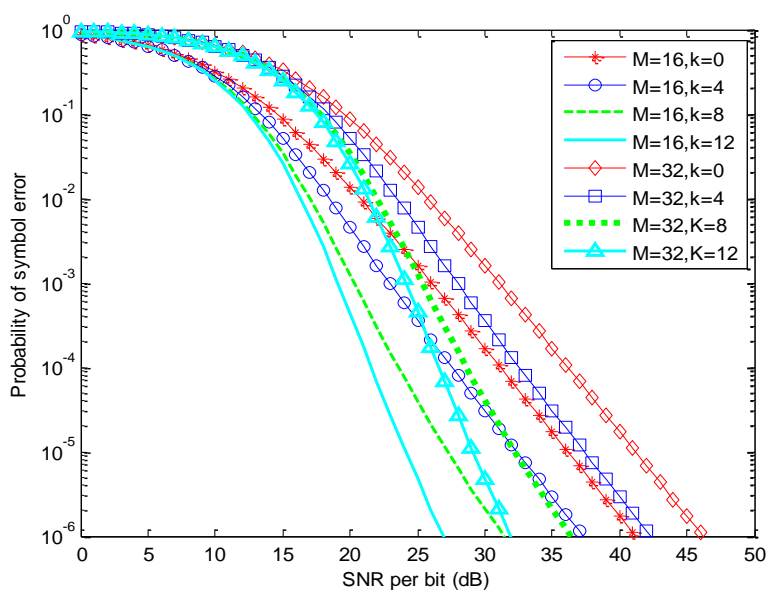

Fig. 4. Approximate SER for M-PSK over Rician fading channels for $N=2$ and for various values of $k$ when $M=16,32$.

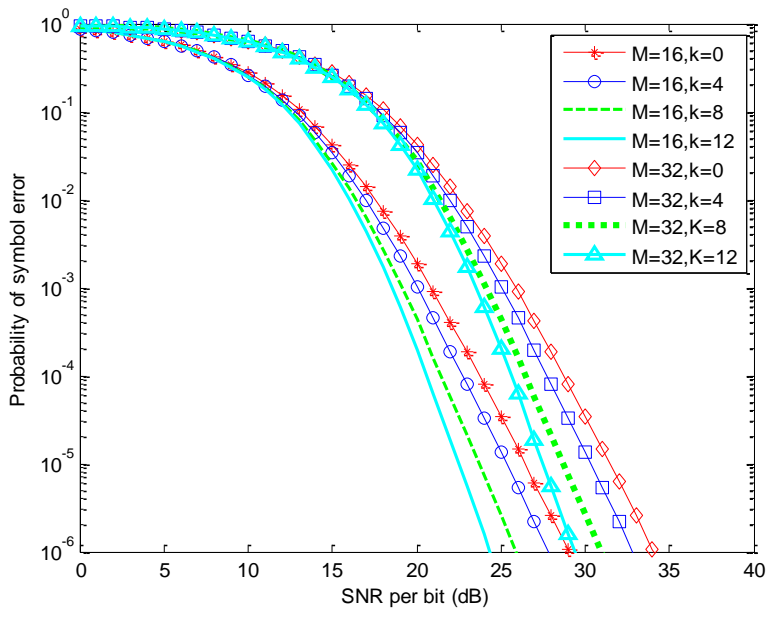

Fig. 5. Approximate SER for M-PSK over Rician fading channels for $N=4$ and for various values of $k$ when $M=16,32$.

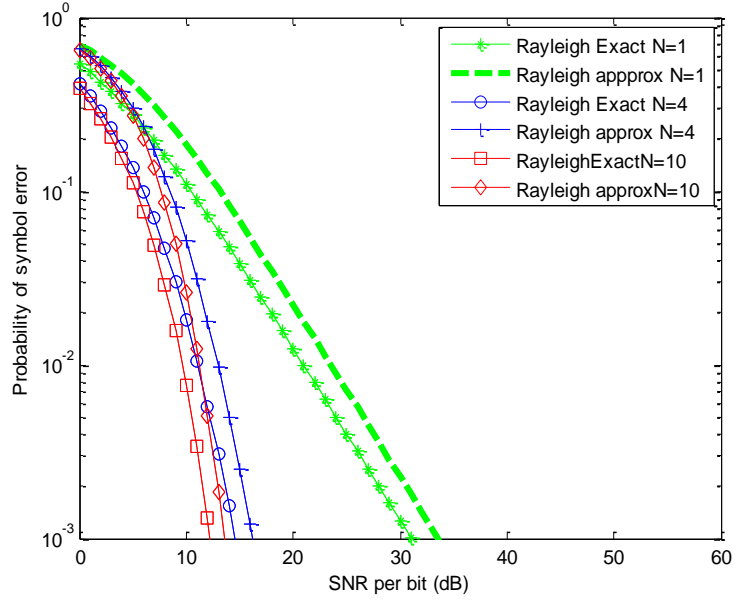

Fig. 6. Exact and approximate SER for M-PSK with space diversity over Rayleigh fading channel for $M=8, N=1, N=4$ and $N=10$.

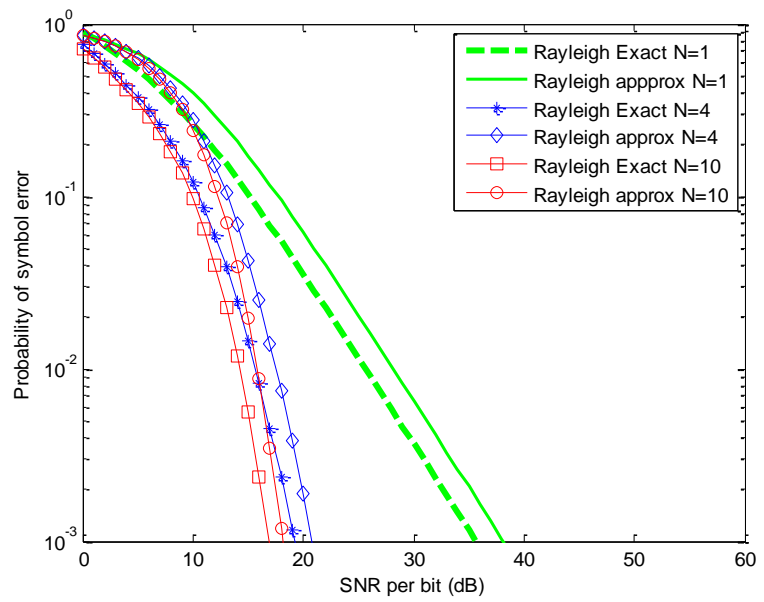

Fig. 7. Exact and approximate SER for M-PSK with space diversity over Rayleigh fading channels for $M=16$ and $N=1, N=4$ and $N=10$.

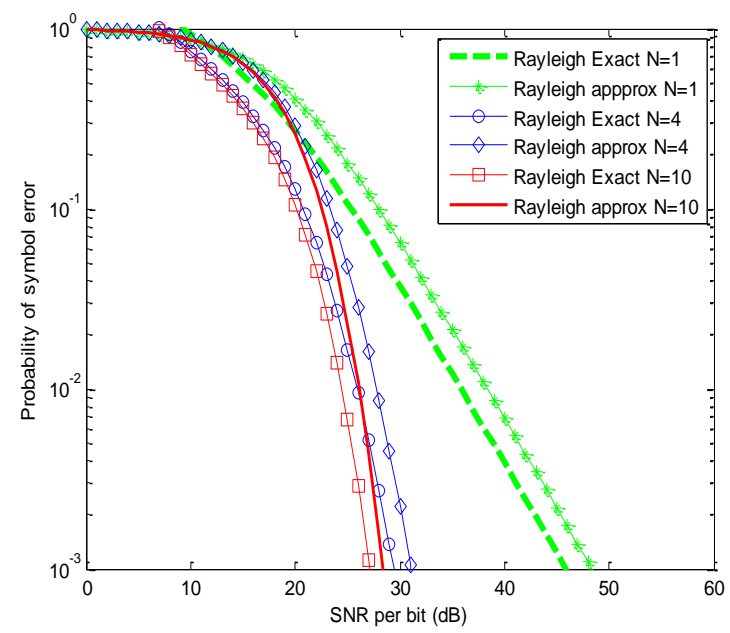

Fig. 8. Exact and approximate SER for M-PSK with space diversity over Rayleigh fading channels for $M=64$ and $N=1, N=4$ and $N=10$. 


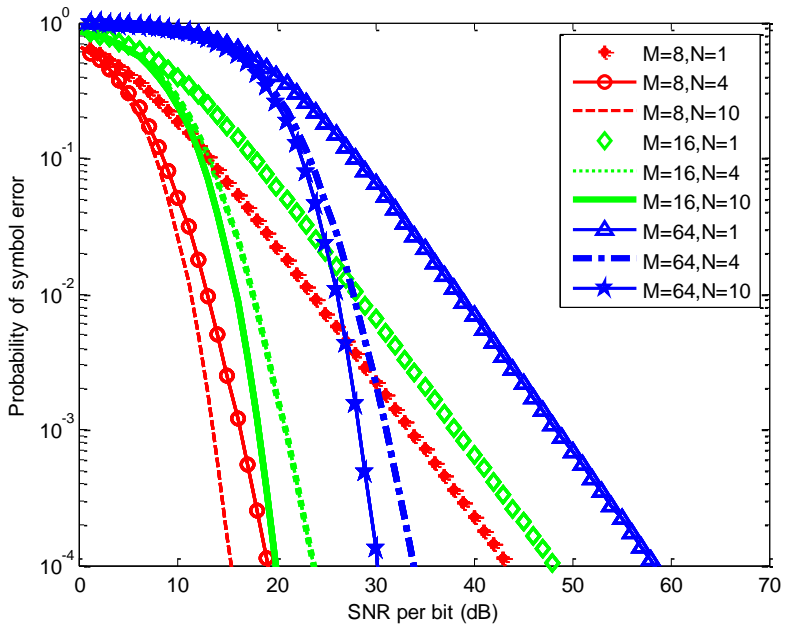

Fig. 9. Approximate SER for M-PSK with space diversity over Rayleigh fading channels for $M=8, M=16$ and $M=64, N=1, N=4$ and $N=10$.

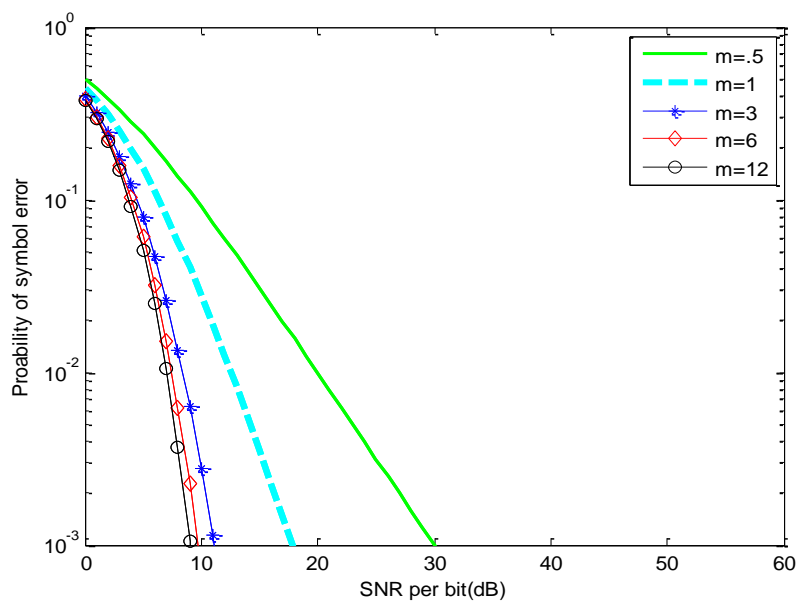

Fig. 10. Approximate symbol error rate for $M$-PSK with STTD over Nakagami- $m$ fading channels when $M=4$ for different values of $m$.

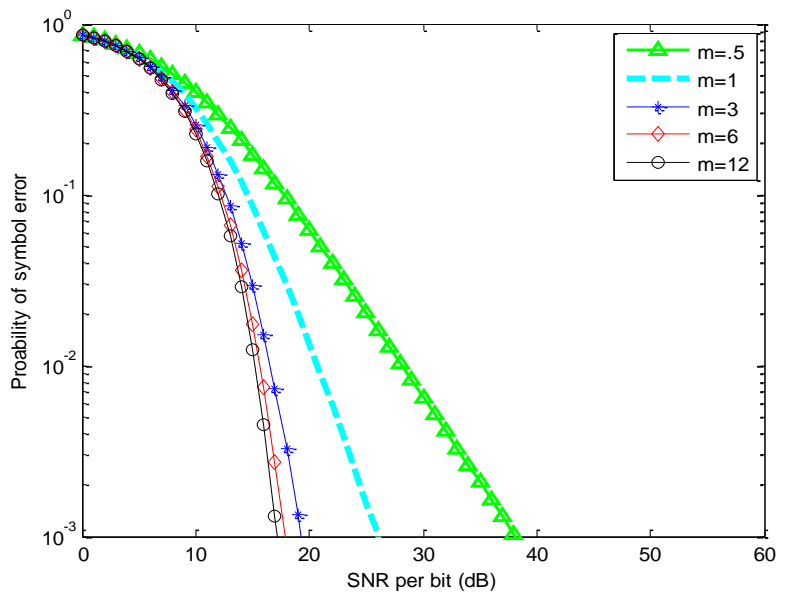

Fig. 11. Approximate symbol error rate for M-PSK with STTD over Nakagami- $m$ fading channels when $M=16$ for different values of $m$.

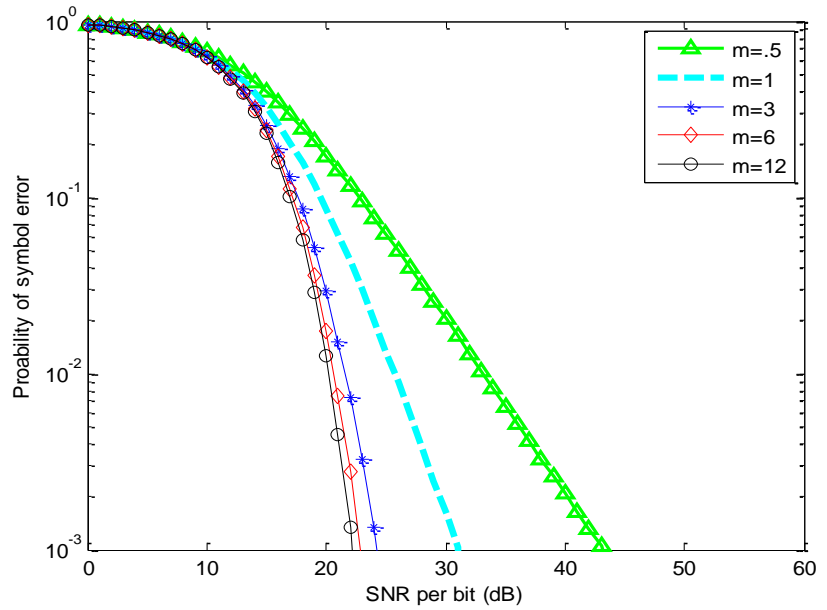

Fig. 12. Approximate symbol error rate for M-PSK with STTD over Nakagami- $m$ fading channels when $M=32$ for different values of $m$.

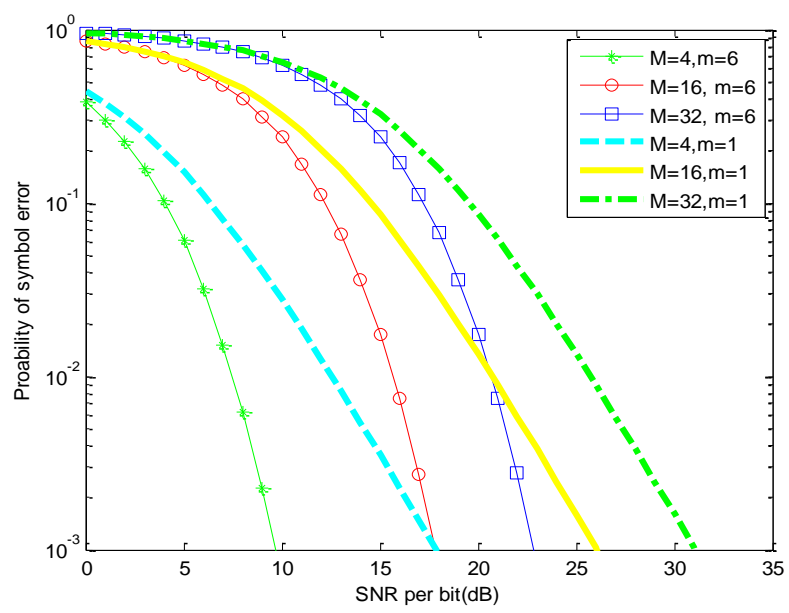

Fig. 13. Approximate symbol error rate for $M$-PSK with STTD over Nakagami- $m$ fading channels when $m=1, m=6$ for different values of $M$.

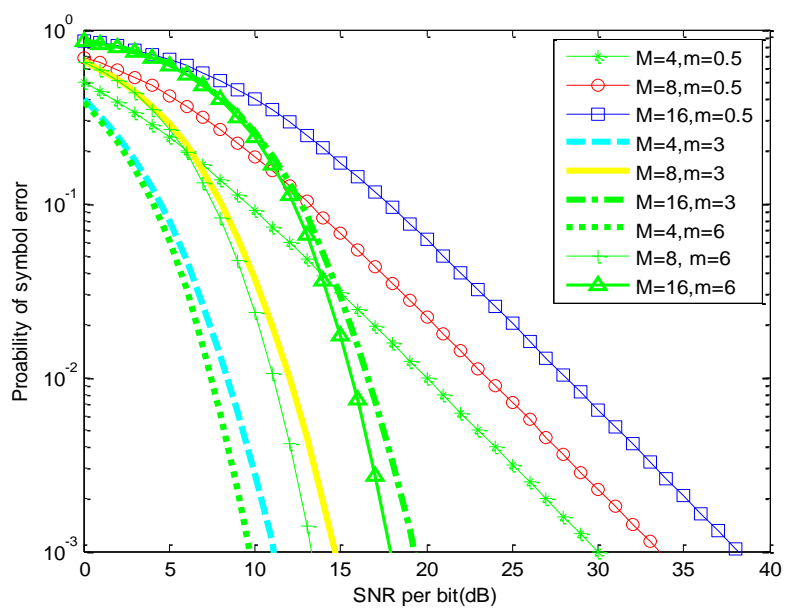

Fig. 14. Approximate symbol error rate for $M$-PSK with STTD over Nakagami- $m$ fading channels when $m=0.5,3$ and 6 for different values of $M$. 


\section{CONCLUSION}

In this study, approximate expressions of the SER for MPSK modulation scheme over i.i.d slow-flat Rician and Rayleigh fading channels using MRC space diversity are derived. In particular, an approximate expression of the SER for M-PSK modulation scheme over a Gaussian channel is used. From the simulation results, one can notice the superior effect of using MRC diversity technique on the overall performance. Moreover, the probability of symbol error with M-PSK modulation scheme over i.i.d slow-flat Rayleigh fading channels for various values of the diversity order $N$ are illustrated. The simulation results show that by increasing the diversity order, the communication reliability will increase. (i.e., as $N$ increases, less power is needed to achieve the same probability of error). Furthermore, comparisons between the exact and approximate SER expressions show that the results are comparable $(2 \% \mathrm{~dB})$. Here, by increasing the Rician parameter, $k$, the overall performance will be improved. However, by decreasing $k$, the worst case scenario can be achieved, i.e., the Rayleigh case when $k=0$. Finally, an approximate expression for the SER for M-PSK over Nakagami- $m$ i.i.d slow-flat fading channel is derived. Moreover, STTD diversity technique is used to enhance the coverage and capacity, where the STTD is applied using two transmit antennas and one receive antenna. From the simulation results, one can observe the effect of increasing the Nakagami- $m$ parameter, $m$, on the overall performance of the model where the fading is less severe in this case. In particular, the overall performance is shown to be the worst when $m=0.5$, and one gets the Rayleigh fading channel case. Furthermore, the SER is lower for higher values of SNR and the performance of the SER is worse for high order PSK modulation scheme.

\section{REFERENCES}

[1] Aniruddha. Chandra, Ananya. Patra, and Chayanika. Bose, "Effect of Imperfect Phase Synchronization on the Error Rate Performance of MPSK in Rayleigh, Rician and Nakagami Fading Channel ", Annual IEEE, India conference (INDICON), 2010.
[2] K. Sivanesan and N. C. Beaulieu, "Exact BER Analysis of Bandlimited BPSK With EGC and SC Diversity in Cochannel Interference and Nakagami Fading", IEEE Commun. Letters, Vol. 8, NO. 10, pp. 623 625, Oct., 2004.

[3] Ahmet. F, Coşkun, and Oğuz. Kucur, "Performance of Joint Transmit and Receive Antenna Selection in Nakagami-m Fading Channels", European wireless conference (EW), pp. 314 - 317, 12-15 April, 2010.

[4] K. Sivanesan and Norman C. Beaulieu, "Precise Performance Analysis of MRC Diversity in Micro-Cellular System with Cochannel Interference", Signal System and Computers, Conference Record of the Thirty-Eighth Asilomar Conference (VTC).

[5] R. Schober, Y. Ma, L. Lampe, and P. T. Mathiopoulos,“ Diversity Combining for Coherent and Differential M-PSK in Fading and Class-A Impulsive Noise ", IEEE Transaction on Wireless Communication, Vol. 4, No. 4, pp. 1425 - 1432, JULY, 2005.

[6] Adel. Ahmad. Ali and Khalid. ALkhudairi, "BER for M-QAM with space diversity and MRC in Rician fading channel", Mobile Computing and Wireless communication International conference (MCWC)., 17-20 Sept. 2006.

[7] K. Sivanesan, Norman. Beaulieu, "Precise Bit Error Rate Analysis of Bandlimited BPSK with EGC and SC Diversity in CCI and Nakagami Fading ",Vehicular Technology conference (VTC), 26-29 September, 2004.

[8] Jian-yun. Zheng, Yi-Sheng. Zhu and Li-ping. Zhu, "Symbol Error Probability Analysis of An FFH/MFSK Receiver with Noise Normalization Combining In a Fading Channel With Partial -Band Jamming", Signal Processing System (SIPS), IEEE Work shop, pp. 377 -381, 17-19 Oct, 2007.

[9] Ronng. He and Junsong. Xie, "BER Performance of M-QAM and MPSK in Nakagami Fading Channel with STTD", IEEE International Symposium on Personal, ln-door and Mobile Radio Communication Proceedings, vol. 2, pp. 1327 - 1330, 2003.

[10] Andrea. Goldsmith, “Wireless Communication”, Cambridge University, 2005.

[11] Upamanyu. Madhow, "Fundamentals of Digital Communication", Cambridge University, 2008

[12] S. Gradshteyn and I. M. Ryzhik, "Table of Integrals, Series and Products", San Diego, Fifth edition, CA: Academic, 1994.

[13] M. Khodeir and M. Jawarneh "Approximate Symbol Error Rate for Mary Phase Shift Keying (M-PSK) using Maximum Ratio Combining (MRC) Technique over Fading Channels", Science and Information (SAI) Conference 2013, London, UK, October 2013 